ever, was slightly colored. Other determinations were made in which the oxalate was precipitated from a cold solution with ammonium chloride present. In this case, a white oxide was obtained. The average of these determinations agreed well with the standard:

Average $\mathrm{Y}_{2} \mathrm{O}_{3}$ in presence of $\mathrm{FeCl}_{3}$, O.I536 gm.; $\mathrm{Y}_{2} \mathrm{O}_{3}$ in standard, O. 1537 gm.

In the Presence of Aluminium.-In order to study the determination of yttrium in the presence of aluminium, Io $\mathrm{cc}$. of a $10 \%$ aluminium chloride solution were added to the $25 \mathrm{cc}$. sample of the standard solution. Yttrium oxalate was precipitated from the cold solution in the presence of ammonium chloride. The result of this determination was also in close agreement with the standard:

Average $\mathrm{Y}_{2} \mathrm{O}_{3}$ in presence of $\mathrm{AlCl}_{3}$, O.I539 gm.; $\mathrm{Y}_{2} \mathrm{O}_{3}$ in standard, O.I537 gm.

In the Prescnce of Lithium.-To the $25 \mathrm{cc}$. of the standard solution of yttrium chloride, ro cc. of a 10\% lithium chloride solution were added. Yttrium was precipitated as in the previous case and with equally satisfactory results:

Average $\mathrm{Y}_{2} \mathrm{O}_{3}$ in the presence of $\mathrm{LiCl}$, O.I536 gm.; $\mathrm{Y}_{2} \mathrm{O}_{3}$ in standard $0.1537 \mathrm{gm}$.

In the Presence of Magnesium.-Yttrium oxalate was precipitated from $25 \mathrm{cc}$. samples of the standard yttrium chloride solution to which ro $\mathrm{cc}$. of a 10\% magnesium chloride solution had been added. As in the preceding cases, there was a complete separation:

Average $\mathrm{Y}_{2} \mathrm{O}_{3}$ in presence of $\mathrm{MgCl}_{2}, 0.1537 \mathrm{gm}$.; $\mathrm{Y}_{2} \mathrm{O}_{3}$ in standard, $0.1537 \mathrm{gm}$.

\title{
Summary.
}

I. Ammonium sebacate affords a quantitative separation of yttrium from sodium.

2. A double precipitation with the same reagent gives a complete separation from potassium.

3. Oxalic acid in the presence of ammonium chloride effects a perfectly satisfactory separation from iron, aluminium, lithium and magnesium. DURHAM, NEW HAMPSHIRE.

[CONtribution from the Biochemical Laboratory of Harvard Medical School.]

\section{IS IONIZATION, AS INDICATED BY CONDUCTIVITY, A NECES- SARY PREREQUISITE FOR THE COMBINATION OF ACIDS WITH BASES?}

By OTro Folin and Fred F. Flanders.

Received March 11, 1912.

Sometime ago a method was described by Folin and Wentworth, based on the titration of acids by means of sodium ethylate, using as solvents 
organic liquids such as chloroform, benzene, toluene, or carbon tetrachloride, and was found satisfactory for the determination of fatty acids. ${ }^{1}$ Since that time a practical method for the determination of benzoic acid has been worked out, ${ }^{2}$ and more recently a method for the estimation of hippuric acid in urin, based on the same principle. ${ }^{3}$

Viewed from the standpoint of the electrolytic dissociation theory, the conditions of these titrations are unusual, and apparently not entirely in accord with the current views.

The reaction between an acid and an alkali is to be regarded as a typical ionic reaction. Walker ${ }^{4}$ states "acidity is, on the theory of electrolytic dissociation, attributed to the presence of hydrion and alkalinity to the presence of hydroxidion in the solution." Therefore the reaction between an acid and an alkali, in its last analysis, must be the reaction between hydrion and hydroxidion.

In organic solvents, however, reactions cannot so easily be regarded as ion reactions. According to Ostwald ${ }^{5}$ substances are not ionized in solvents of the character of benzene. He says, "In order therefore to separate a substance from its solution in water by ether, benzene, or any such liquid, it must be brought into a condition in which it is neither an ion itself, nor a constituent of one." "It is only the non-ionized portion which is affected by the process of extraction."

From this point of view then, an acid could only be present in solution in benzene in the unionized state. This idea is confirmed, in so far at least as benzoic acid in benzene is concerned, by Walker ${ }^{6}$ who gives the molecular weight of benzoic acid, determined by the freezing-point method in benzene, as $223-236$, showing that it corresponds nearly to double molecules. Many investigators ${ }^{7}$ have shown that substances are ionized to a far less degree in alcohol than in water. According to Kablukoff ${ }^{8}$ the ionization decreases as the molecular weight of the alcohol increases. The same investigator finds that even hydrochloric acid is only very feebly ionized in such solvents as benzene, toluene and xylene. ${ }^{9}$

Furthermore, that there is a certain parallelism between ionization and

${ }^{1}$ Folin and Wentworth, J. Biol. Chem., 7, 42 I (Igro).

${ }^{2}$ Folin and Flanders, J. Am. Chem. Soc., 33, I6 (191 I).

${ }^{3}$ Folin and Flanders, J. Biol. Chem., I I, 257 (1912).

4 "Introduction to Phys. Chem.," p. 315, 5th ed.

"Ostwald, "Sci. Foundations of Anal. Chem.," 3d Eng. ed., p. 94. See also p. 56 for similar statements.

'Walker, "Elements of Phys. Chem.," 5th ed., p. 205.

'Fitzpatrick, Phil. Mag., 24, 378 (1887). Hartwig, Wied. Ann., 33, 58 (1888); 43, 838 (1891). Völlmer, Ibid., 52, 328 (1894). Kawalki, Ibid., 52, 324 (1894). Schall, Z. physik. Chem., I4, 70r (1894). Kablukoff, Ibid., 4, 429 (I898). Jones, Ibid., 3I, 133 (1899).

${ }^{8}$ Z. physik. Chem., 4, 432 (I889).

Ibid., 4, 429 (1889). 
chemical reactivity on the one hand and chemical reactivity and conductivity on the other, seems to be a fundamental concept of the theory of electrolytic dissociation. Jones ${ }^{1}$ says "Molecules as such have little or no chemical affinity;" and "We have already reached a point where we can say that nearly all, if not all chemical reactions are due to ions, molecules as such not entering into chemical action."

Ostwald ${ }^{2}$ outlines a certain experiment, "To show the parallelism between conducting power and capability of chemical reaction."

The failure of dry hydrogen sulfide ${ }^{3}$ to precipitate mercuric chloride in absolute alcohol has been cited as due to lack of ionization. ${ }^{4}$ From these various expressions, one is led to the conclusion that a solution of a substance, to possess chemical reactivity, must be ionized, and that this ionization would be disclosed by its conductivity. Or conversely, a substance which did not exhibit some degree of conductivity would appear to be non-ionized and hence would ordinarily not be expected to possess chemical reactivity. Kahlenberg, however, has never accepted the electrolytic dissociation theory and from among his many ingenious experiments, made to show that it does not hold for reactions in organic solvents, may be mentioned the reaction of dry hydrogen chloride on dry copper oleate in benzene solution. ${ }^{5}$

Our experimental evidence proves that all classes of acids may be titrated by sodium ethylate, in organic solvents. The only limitation seems to be that of solubility of the acid in the solvent employed. Acids which were not readily soluble in chloroform were first dissolved in $10 \mathrm{cc}$. of absolute alcohol, diluting to roo cc. with chloroform. Oxalic, succinic, malic, citric and tartaric acids were treated in this manner and found to give sharp end points. Hydrochloric, acetic, propionic, caproic, cinnamic, lactic, salicylic, benzoic and picric acids were dissolved in chloroform alone. The end points were excellent. Even picric acid yielded quantitative results. Particularly, the very weak acids which cannot be titrated at all in aqueous solution on account of hydrolysis, are found to yield excellent results in organic solvents. This point has been tested on stearic, palmitic and oleic acids, and even on hydrogen sulphide. The latter, dissolved in chloroform, titrates with a fairly sharp end point when phenolphthalein is employed as indicator. Carbon dioxide could

1 "Elements of Phys. Chem.," p. 440, 4th ed.

2 "Sci. Foundations of Anal. Chem.," 3d Eng. ed., p. 236.

${ }^{3}$ Jones, "Elements Phys. Chem.," p. 439.

- Purely as a matter of interest, we have found that mercuric chloride dissolved in equal parts of absolute alcohol and benzene can be titrated with sodium ethylate giving a fair end point with phenolphthalein. The titration appears to be nearly quantitative.

s Kahlenberg, J. Physic. Chem., 6, I (Igoz). See also Stieglitz, "Qual. Anal.," Vol. I (I9II), pp. 72 to 87 , for a most interesting discussion of this phase of the subject. 
not be titrated either in chloroform or in benzene. The reason for this is obvious, carbon dioxide in the absence of water not being an acid.

In view of the apparent discrepancy between our own observations and the accepted teachings it seemed to us well worth while to determin the conductivity of some of our solutions. The results obtained have, however, not cleared up this discrepancy, as shown by the specific conductivities recorded below:

Solution.

o. I $N$ sodium ethylate.....

$0.05 N$ sodium ethylate....

o. I $N$ benzoic acid.

$0.05 N$ benzoic acid.........

Sodium benzoate
(Prepared from sodium and absolute alcohol)

(o.I $N$ sodium ethylate diluted with an equal volume of benzene)

In pure benzene

(o.r $N$ benzoic acid in benzene diluted with an equal volume absolute alcohol)

(Saturated soln. in equal parts absolute alcohol and benzene)
Specific conductivity at $18^{\circ}$.

$1.4 \times 10^{-9}$

I. $6 \times 10^{-4}$

Too small to tneasure

$8.6 \times 10^{-7}$

$2.8 \times 10^{-2}$

As was to be expected, the conductivity of the benzoic acid in benzene was immeasurably small.

The benzoic acid solution, whether in a mixture of alcohol and benzene or benzene alone, could be titrated with a perfectly sharp end point, either by the sodium ethylate or the same diluted with an equal volume of benzene. The presence of benzene considerably decreases the conductivity of the solutions, but not the reactivity, as the titrations were as sharp as when the larger proportions of alcohol were used. The sodium benzoate is not very soluble in the mixed solvent, and is partially precipitated toward the end of the titration.

In order to avoid this difficulty, a solution of oleic acid was prepared for study. Sodium amylate was used as the alkali, as, according to Kablukoff, ${ }^{1}$ it might be expected to have much less conductivity than the ethylate. The following table gives the conductivities of the solutions employed:

Solution.

o. I $N$ sodium amylate...... In pure amyl alcohol

$0.05 N$ sodium amylate.... (A new soln. of amylate in equal parts amyl alcohol and benzene)

o. I $N$ oleic acid.......... In pure benzene

$0.025 N$ sodium oleate..... (In equal parts amyl alcohol and benzene)

In pure amyl alcohol
Specific conductivity at $18^{\circ}$.

$2.2 \times 10^{-6}$

$3.3 \times 10^{-7}$ Too small to measure

$2.5 \times 10^{-7}$

$3.8 \times \mathrm{ro}^{-7}$

$4.5 \times \mathrm{ro}^{-7}$

Pure amyl alcohol.......... ...........

2 Loc. cit. 
The amyl alcohol itself had some conductivity, as is shown by the figure on the pure solvent. It, as well as the benzene, had been treated with sodium and distilled.

Titrations were made with the $0.05 N$ amylate in mixed amyl alcohol and benzene, against the oleic acid in pure benzene, and also by adding an equal volume of amyl alcohol before titrating. The end points were sharp and satisfactory. The $0.025 N$ sodium oleate was prepared by titrating a measured amount of oleic acid in benzene, to which had been added an equal volume of amyl alcohol, with the $0.05 \mathrm{~N}$ amylate in $50 \%$ benzene solution.

The table shows one interesting feature. With the exception of the first, the conductivities of the solutions appear to be almost independent of the dissolved substance; that is, they are of about the same order as the amyl alcohol itself and are possibly due to it in each case. The first solution of sodium anylate in alcohol had been prepared from alcohol which had not been treated with sodium and distilled, and its greater conductivity is probably due to this fact. Titrations were made with both this solution and that from purified alcohol mixed with benzene. No difference could be observed in speed of titration or sharpness of the end point.

Other indicators than phenolphthalein were also tried, with the oleic acid and sodium amylate, both made up in a mixture of equal parts of amyl alcohol and benzene. Phenolphthalein, thymophthalein, rosolic acid, alizarin, hematoxylin aud alizarin red gave results as regards sharpness of end point, about in the order named. Any one of the first three named might be used with a fair degree of success. Phenolphthalein is, however, the most satisfactory. Other indicators, congo red, methyl orange, eosin, fluorescein, tropaeolin oo, and dimethylaminoazobenzene were tried. None of these gave the acid reaction with oleic acid. From this it appears that the distinction between mineral and other acids holds here as in aqueous solutions. A trace of mineral acid shaken with the solution gave the acid reaction with all the indicators.

The conductivity measurements recorded in this paper were made with the Ostwald cell, with plates set about $0.5 \mathrm{~cm}$. apart. Unusual accuracy is not claimed for the measurements. The resistance of the solutions is very high, and accurate measurements would require apparatus especially suited to this class of work. We are not prepared to assert that our solutions were absolutely free from water, in the sense used in physical chemistry, nor to deny that the results which we obtain on titrating weak acids in organic solvents, can be explained on the basis of the enormous speed of a few ions which may be present without appreciably affecting the conductivity of the solutions. ${ }^{1}$ It is of course a

${ }^{1}$ Haber, Z. Elektrochem., ro, 433, 473 (I904). 
fact that we have always had some alcohol present in our titrations, as carrier of the alcoholate, and even amyl alcohol has some dissociating power. Curiously enough, however, the addition of alcohol in larger amounts makes the end point of the titrations less sharp than when the amount of alcohol is kept at a minimum. We would emphasize that our titrations have been made with solutions of acid and alkali which have almost no conductivity, that the resulting salt solutions also have practically no conductivity, that the reaction is instant and that the action of the indicator does not differ materially from that observed in aqueous solutions.

[Contributions from the Research Laboratory of Physical Chemistry of The Massachusetts Instittete of Techiology. No. 82.]

\section{THE DISSOCIATION PRESSURES OF SODIUM AND POTASSIUM HYDRIDES.}

BY FREDERICK G. KEYES.

Received March 23, 1912.

\section{Review of Previous Investigations.}

Gay-Lussac and Thénard ${ }^{1}$ were the first to observe that metallic sodium and potassium absorb hydrogen. In the case of sodium no quantitative results were obtained. They observed, however, in the case of potassium, that one equivalent of the metal absorbed one-quarter of an equivalent of hydrogen.

Troost and Hautefeuille ${ }^{2}$ studied the reaction and measured the equilibrium pressure of hydrogen over both the metals. They also attempted to determin the composition of the hydrides. The analysis was made by saturating a definit weight of metal and subsequently pumping out and measuring the hydrogen gas. No details of the experiments are given, but the formulas $\mathrm{Na}_{2} \mathrm{H}$ and $\mathrm{K}_{2} \mathrm{H}$ are assigned. Equilibrium measurements for both hydrides were made from $330^{\circ}$ to $430^{\circ}$. The values will be presented below.

Henri Moissan ${ }^{3}$ prepared both hydrides by heating the metals in a current of hydrogen gas. The excess of metal was then extracted from the product by means of liquid ammonia. The analysis of the hydrides thus obtained was made by decomposing the compounds with water and measuring the volume of gas evolved. The data gave the formulas $\mathrm{NaH}$ and $\mathrm{KH}$. Moissan studied various properties of the hydrides. He found that they were insoluble in organic solvents, and that they reacted with carbon dioxide to form formates. The dissociation pressures were not measured.

${ }^{1}$ Recherches physico-chimiques, $\mathrm{I}, \mathrm{I} 76$.

${ }^{2}$ Compt. rend., 78, 807 (1874).

${ }^{3}$ Ibid., I34, 7 I (1902). 